\title{
Bewährtes mit Neuem verknüpfen
}

- Das Jahr neigt sich dem Ende zu, die Tage werden kürzer. Zeit, ein Resümee zu ziehen und Pläne für die Zukunft zu schmieden! Genauso hielten es die Teilnehmer unseres 3. ergotags, der Anfang Oktober in Leinfelden bei Stuttgart stattfand. Ein Tag, der zweierlei geschickt miteinander verknüpfte: einerseits theoretische Vorträge, um altes Wissen aufzufrischen und Neues $z u$ lernen. Andererseits praktische Vorträge mit bewährten, aber auch zukunftsweisenden Ideen und Ansätzen.

_ Der ergotag machte Mut, auch mal die traditionellen Trampelpfade zu verlassen. Zusätzlich neue Wege zu erobern. Denn: Unser Aufgabengebiet wird zunehmend komplexer! Wir arbeiten verstärkt interdisziplinär, beraten Eltern oder Lehrer, und wir tragen durch unsere neu entwickelten Angebote die politische Vorgabe „Prävention vor Therapie“ konsequent mit!

_ Das Konzept des ergotags ist auch in diesem Jahr aufgegangen - die befragten Besucher loben die Vielschichtigkeit der Vorträge und die Mischung aus Wissenschaft und Praxis, aus Forschung und Therapie. Ganze 96 Prozent würden den ergotag weiterempfehlen, was uns natürlich besonders freut!

_ Auch unsere neue Fachzeitschrift neuroreha verknüpft Forschung und Therapie. Jedes Heft widmet sich einem Schwerpunktthema aus der Neurorehabilitation und versorgt Sie mit aktuellen Erkenntnissen, welche Sie direkt in Ihre Therapie einfließen lassen können. Lesen Sie gleich die erste Ausgabe kostenlos Probe. Überzeugt? Dann bestellen Sie die neuroreha noch heute als ergopraxis-Abonnent zum Vorzugspreis von 85 Euro pro Jahr!

_Ziehe ich ein persönliches Resümee des vergangenen Jahres, machen mich Ihre positiven Rückmeldungen zu unseren Artikeln sowie zu unseren Beilagen ergopraxis.Refresher und praxisprofi besonders froh. Ihr Lob spornt uns an, auch im nächsten Jahr neue Ideen für Sie - unsere Kollegen - zu entwickeln. Ihre Anregungen beflügeln uns, Sie auch im nächsten Jahr mit brandaktuellen, fundierten und praxisnahen Themen zu versorgen!

Im Namen der gesamten ergopraxis-Redaktion wünsche ich Ihnen eine „staade“ Zeit, einen beschwingten Rutsch ins neue Jahr - und natürlich viele kreative Ideen für 2010 und eine große Portion Energie, diese umzusetzen!

Ihre

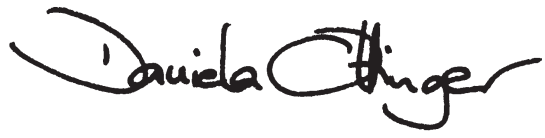

